\title{
The Characteristics of Taiwan Persuasive Maps Made by Japanese
}

\author{
Ching-Jen $\mathrm{Kao}^{\mathrm{a}, *}$ \\ ${ }^{a}$ Chinese Culture University, Department of Geography, chingjen1958@gmail.com \\ * Corresponding author
}

Keywords: metaphor, rhetoric, aesthetics, propaganda

\begin{abstract}
:
Since Harley (1989) proposed his concept of deconstructing the map, a new thinking about map is established and cause some cartographers rethinking the definition of map and shift their scientific axioms to evaluate maps. Persuasive maps are typically designed differently than scientific visualization, which are introduced more than 200 years represented for a given situation, especially to elicit particular interpretation. During 1895-1945 Taiwan was ruled under Japan. The Empire was enthusiastic in set up infrastructure including triangulation which brought Taiwan into the era of Modern Maps. Besides, some thematic maps such as the beautiful Ukiyo with bird's eye view were made. Nowadays these maps are all digital as cartographic heritage, they provide as research materials. But most of the studies discuss these maps from historical point of view, and less metaphor of cartography were proposed. According to the stratified sampling strategies, twenty-one maps are selected to discuss in this study. The contents analysis items are following the discussions of Tyner (1982,2018) and Muehlenhaus (2011). These maps could divide into four kinds. The first one is the cartoon and humorous maps, which emphasize the Japanese was a strong giant, while Taiwanese and Manchurian were weak. This kind of maps seems not a real map, but their sensational impact full of persuasion. The second kind of maps is unique skill with bird's eye view landscapes. Most of these maps and posters were presented in the Exposition of Colonial Administration for Forty Years in Taiwan. The main goal of this kind of maps were intended to show off Japanese colonial achievements, for Japan had established many infrastructures in Taiwan especially built the railways around the island, policies Following the policies of Japanese Empire, government of Taiwan encourage people enjoy travel to promote economy and tourism. The techniques of this kind of maps were transferred from Ukiyo and western perspective concept. Realistic and colorful symbols make this kind of maps looked vivid and attractive as well as easy to read, created a succinctly communication and eliciting the happy journey imagination. The third kind of maps is so called authoritative map. They were created during the early colonized days. In that time controlling and managing Taiwan indigenous was Japan urgent policy. Based on the topographic maps, through the red lines or red circles to attract the eyes attention and clearly suggest the troop's planning routes. No extra information, color play the main role. The last one kind of maps is same as the third one, but their issues were focused on war. Various projections cause different distortions which could highlight the topic but not really the truth. While some maps omit parallels and meridians to confused the reader's location perception. Even though ship and airplane were the common used symbols, their dynamic association east to direct the battle condition. Different color used to distinguish the safety level. Especially, in all kinds of these maps, Japan and its colonies always showed in red. The reason not only because red easy to attract eyesight but also red is Yamato favorite color. These persuasive maps persuade us that in the colonized era Japanese really provided great contribution to Taiwanese cartographic history.
\end{abstract}

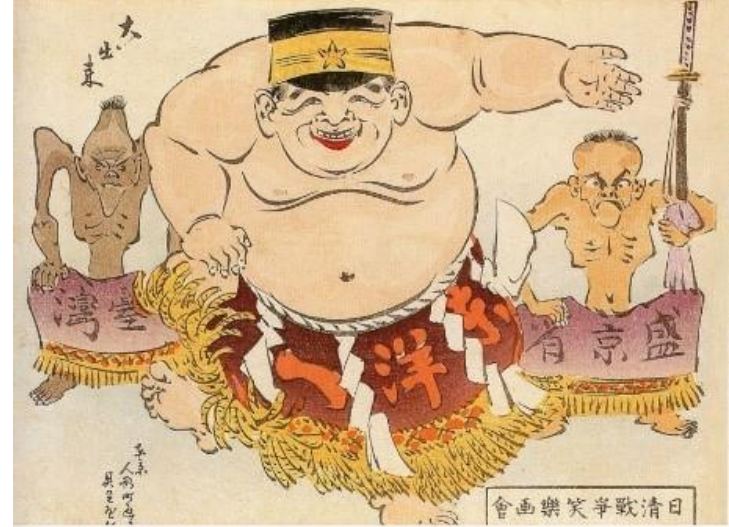

Figure 1. :Full of sarcastic cartoon map

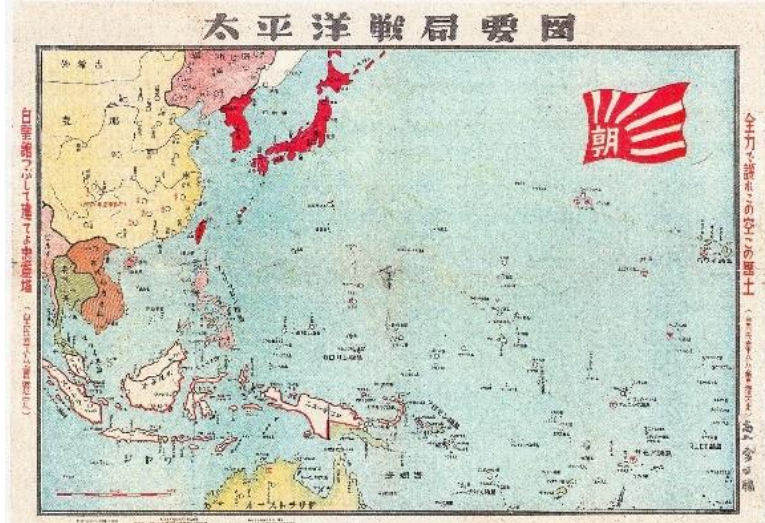

Figure 2. :Authoritative map 